Review Article

\title{
Cholangiocarcinomas: New Insights from the Discovery of Stem Cell Niches in Peribiliary Glands of the Biliary Tree
}

\author{
Vincenzo Cardinale, ${ }^{1}$ Maria Consiglia Bragazzi, ${ }^{1}$ Guido Carpino, ${ }^{2,3}$ Alessia Torrice, ${ }^{1}$ \\ Yunfang Wang, ${ }^{4}$ Lola McAdams Reid, ${ }^{4}$ Eugenio Gaudio, ${ }^{2}$ and Domenico Alvaro ${ }^{1,5}$ \\ ${ }^{1}$ Department of Medico-Surgical Sciences and Biotechnologies, Polo Pontino, Sapienza University of Rome, Rome, Italy \\ ${ }^{2}$ Department of Anatomical, Histological, Forensic Medicine and Orthopedics Sciences, Sapienza University of Rome, Rome, Italy \\ ${ }^{3}$ Department of Movement, Human and Health Sciences, Division of Health Sciences, University of Rome "Foro Italico", Rome, Italy \\ ${ }^{4}$ Department of Cell Biology and Physiology, Program in Molecular Biology and Biotechnology, \\ University of North Carolina School of Medicine, Chapel Hill, NC 27599, USA \\ ${ }^{5}$ Eleonora Lorillard Spencer-Cenci Foundation, Rome, Italy
}

Correspondence should be addressed to Domenico Alvaro; domenico.alvaro@uniromal.it

Received 11 December 2013; Revised 23 February 2014; Accepted 17 March 2014; Published 24 April 2014

Academic Editor: Mustapha Najimi

Copyright (C) 2014 Vincenzo Cardinale et al. This is an open access article distributed under the Creative Commons Attribution License, which permits unrestricted use, distribution, and reproduction in any medium, provided the original work is properly cited.

Peribiliary glands (PBGs) are located in the large intrahepatic and extrahepatic bile ducts. Although they were described many years ago, their functions have been elucidated only in the last couple of years when our group demonstrated that PBGs are niches of multipotent stem/progenitor cells of endodermal origin. These cells express genes of multipotency and can be rapidly differentiated in vitro into hepatocytes, cholangiocytes, and endocrine pancreatic cells. PBGs share common features, in terms of stem/progenitor cell niches, with pancreatic duct glands and colon crypts, glandular structures representing in the adult life the endodermal remnants of fetal life. PBG stem/progenitor cells participate in the renewal of surface biliary epithelium and are active players in chronic pathologies of the biliary tree as well as in cholangiocarcinomas (CCA). Specifically, a large amount of recent evidence indicates that the pure mucin-CCA originates from PBGs; this could explain the similarities with pancreatic ductal adenocarcinoma and colorectal cancer, which also originate from transformed gland cells. In this paper, we summarized our recent findings concerning structure and functions of PBGs with the implications for liver pathophysiology and, specifically, for cancers of the biliary tree.

\section{Introduction}

Although peribiliary glands (PBGs) have been described for many years, their functions were clarified only a couple of years ago when our group demonstrated that niches of multipotent stem/progenitor cells of endodermal origin are located at the bottoms of these glands. This observation and subsequent research from our group are highlighting a relevant role of PBGs in liver pathophysiology with important implications for the renewal of biliary epithelium and liver parenchyma as well as for the pathogenesis of chronic liver diseases and liver cancers. In this paper we summarize our recent findings in the area of PBGs with a specific focus on the implications for the origin and pathogenesis of cholangiocarcinoma (CCA).

\section{Anatomy and Physiology of Peribiliary Glands}

The biliary tree is composed of intrahepatic (IH) and extrahepatic (EH) bile ducts lined by epithelial cells called cholangiocytes. For many years, in humans and in other animal species [1] (mouse, rat, pig, monkey, dog, pig, cow, sheep, and rabbit) glandular elements along the biliary tree have been described and named peribiliary glands (PBGs) 
[2] (Figure 1). PBGs are tubular-alveolar structures composed of serous and mucinous acini located in the $\mathrm{EH}$ and large IH bile ducts [3] (Figure 1). We recently performed a careful morphological and morphometric analysis of PBG in adult and fetal human biliary tree [4-6] (Figure 2). PBGs are located in the deeper tissue of the bile duct walls with some lobules found outside the bile duct walls (Figure 1). The PBGs communicate with the lumen through ducts that open into the diverticula [3]. In the intrahepatic bile ducts (IHBDs), PBGs start at the level of septal bile ducts where PBGs can be observed occasionally as small evaginations of the bile duct epithelium, with the interlobular bile ducts being devoid of such glands [4] (Figure 3). PBGs become significantly more dense at the level of the large (area and segmental) IH ducts. In the $\mathrm{EH}$ biliary tree, $\mathrm{PBG}$ are present along the entire lenght of the bile ducts, including the hepato-pancreatic common duct (Figure 3). In large IHBDs and $\mathrm{EH}$ bile ducts (EHBDs) two types of glands were observed: intramural and extramural PBGs. The intramural PBGs are positioned inside the duct wall and appear as shallow evaginations of the bile duct epithelium. Extramural PBGs are located outside the wall and are composed of PAS-negative cells.

The quantitative morphometric analyses have demonstrated how, in the human adult biliary tree, PBGs are in high numbers in branching points such as the cystic duct and perihilar and periampullary regions [5]. Gallbladder is devoid of PBGs, whereas the cystic duct wall has the same histological features found elsewhere in the EHBDs [2-5].

For many years the functions of PBGs remained virtually unknown. Glandular epithelial cells play a role in the mechanisms of bile concentration by reabsorbing bile constituents that are then drained by surrounding vessels and lymphatics [2,3]. These glands show some secretory activities including secretion of mucinous substances and are also positive for pancreatic digestive enzymes [7]. For many years, studies in some invertebrate fish have indicated a functional heterogeneity of biliary tree cells including a role as putative pancreatic cells controlling glucidic homeostasis [8].

2.1. Stem/Progenitor Cells in Human PBGs. Compelling evidences, such as the presence of pancreatic markers, both in the embryo and adult $[9,10]$, suggest that PBGs contain precursor populations that are remnants of endodermal stem cell proliferation at the porta hepatis and give rise to the developing hepato-biliopancreatic bud. Indeed, the extrahepatic PBGs arise as a few small saccular evaginations that branch into the surrounding mesenchyma from the forming EHBDs $[11,12]$. Postnatally, the PBGs are dispersed throughout the thickness of the bile duct. With maturation to adulthood, the bile ducts lengthen resulting in location of the PBGs in discrete clusters especially at the branching points of the ducts $[5,13,14]$. The number of glandular acini reaches a maximum within the first year of life, thereafter remaining remarkably constant in adult life. The common bile duct is functionally active with respect to the bile flow starting from the 12th week of gestation [12]. In the adult, we investigated different portions of the biliary tree obtained from donor livers, donor pancreata, or tissues discharged from major liver surgery

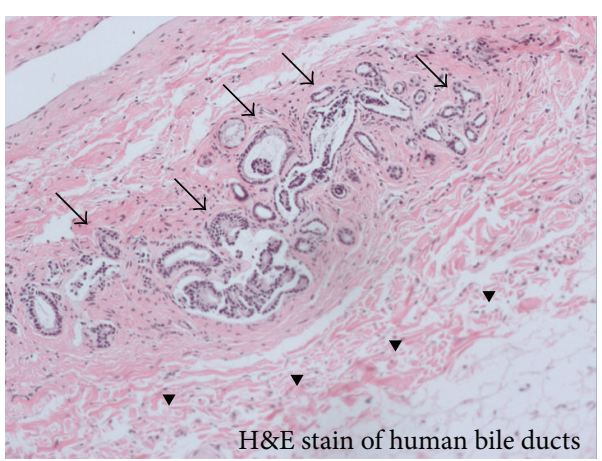

FIgURE 1: Peribiliary gland histology. Hematoxylin and eosin stain (H\&E stain) demonstrating the presence of PBGs within the wall of the common bile duct of normal adult liver. PBGs (arrows) are tubular-alveolar structures composed of serous and mucinous acini located in the extrahepatic and large intrahepatic bile ducts. PBGs are located in the deeper tissue of the bile duct walls near fibromuscular layers (arrow heads) with some lobules found outside the bile duct walls. The PBGs communicate with the lumen through ducts that open into the diverticula. Magnification (M) 10x.

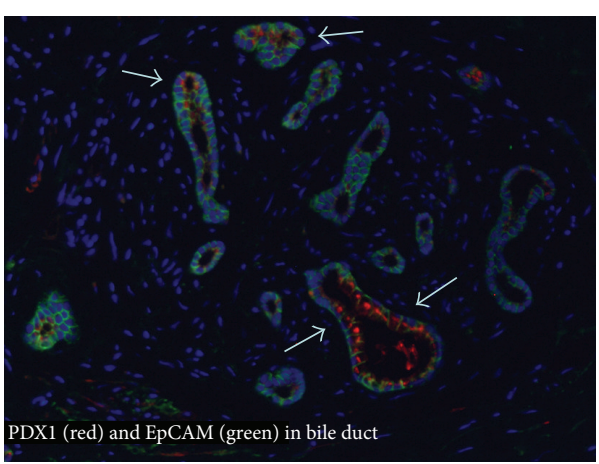

FIGURE 2: Biliopancreatic stem/progenitors in peribiliary glands of adult liver. The immunofluorescence demonstrated stem cells deeper inside of the wall, co-expressing endodermal nuclear transcription factors essential for liver and pancreas formation (e.g., PDX1 in red) together with endodermal stem cell markers (e.g., EpCAM in green) (arrows).

[2, 4]. Multipotent stem/progenitor cells of endodermal origins have been found in PBGs throughout bile ducts and shown able to differentiate toward hepatocytes, cholangiocytes, or pancreatic islet cells. We have yet to assess if these stem cells can give rise to pancreatic acinar cells. The cells have been named human biliary tree stem/progenitor cells (hBTSCs). Our data suggest that they are precursors of stem/progenitors cells located in ductal plates of fetal and neonatal livers and in canals of Hering of pediatric and adult livers. Therefore, the stem/progenitors cells located in PBGs represent an extant trace of the common embryological origins of liver, biliary tree, and pancreas. Although pancreatic committed progenitors located within pancreatic duct glands have long been known [15-17], the biliary tree stem cell populations include the first pancreatic stem cell subpopulations identified postnatally and persisting into adulthood [2, 4, 5, 18] (Figure 2). Particularly high numbers 


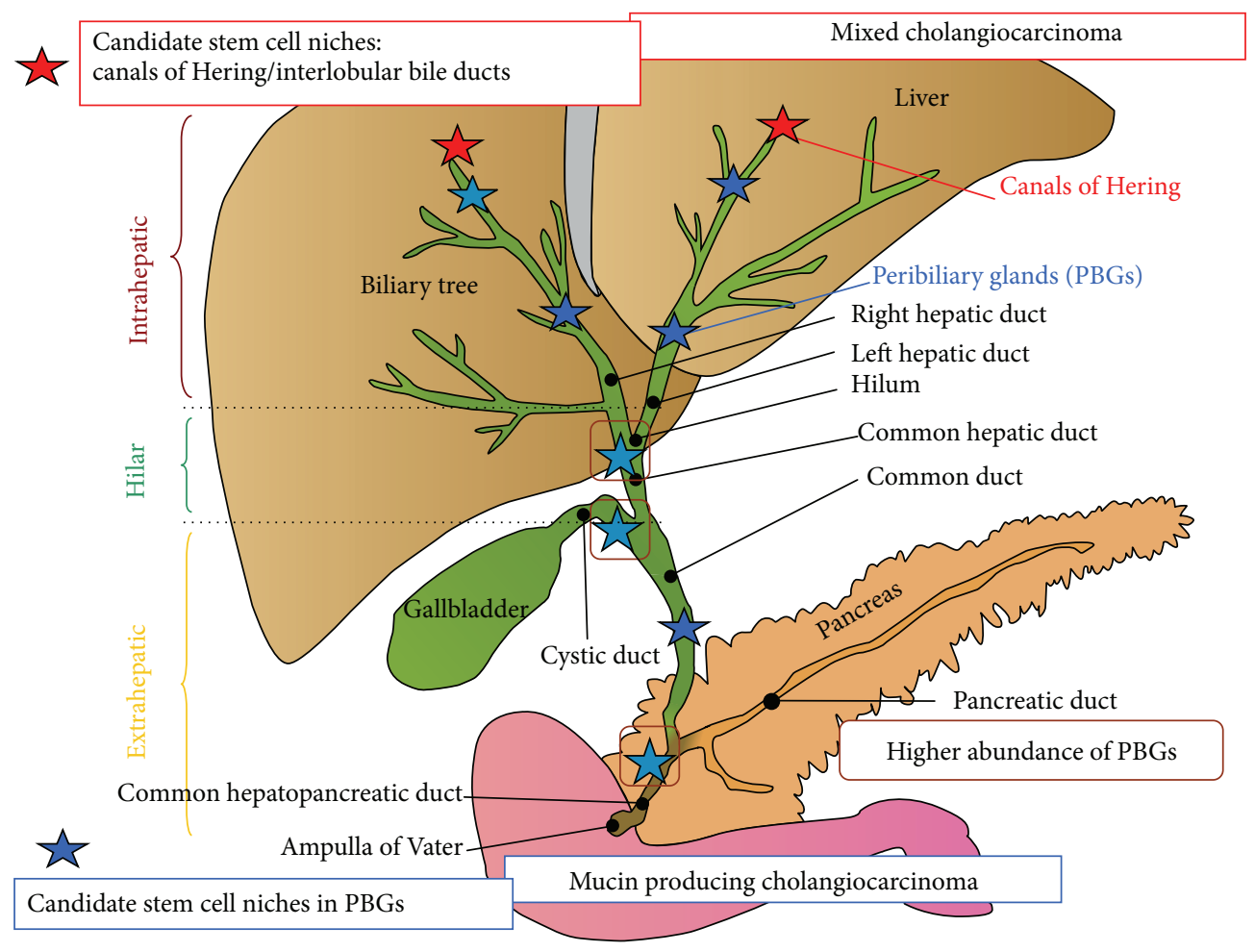

FIGURE 3: Liver stem cell niches and cholangiocarcinoma classification based on cells of origin. Hepatic stem cell niches containing human hepatic stem cells (hHpSCs) (red stars) are located in adult liver, within the canals of Hering or in activated interlobular bile ducts. Biliary tree stem cell niches containing human biliary tree stem/progenitor cells (hBTSCs) (blue stars) are located in peribiliary glands (PBGs). PBGs start at the level of septal intrahepatic bile ducts where glands can be observed occasionally as small evaginations of the bile duct epithelium, with the interlobular bile ducts being devoid of glands. In the extrahepatic biliary tree, PBGs are present along the entire bile ducts, including the hepato-pancreatic common duct. Gallbladder is devoid of PBGs, while cystic duct wall has the same histological features of the other extrahepatic bile ducts. The existence of two different stem cell compartments and the associated cell lineages may result in multiple cells of origin of cholangiocarcinoma (CCA). Mixed-CCA could be considered the CCA subtype originating from hHpSC-derived lineages. Mucin IH-CCA, perihilar CCA, and distal EH-CCA could be considered the CCA subtypes originating from hBTSC derived lineages. The figure is a modification (with permission) of a figure prepared by Ms. Gemma Mendel for the review article referenced as [44].

of the pancreatic stem cell subpopulations are found within the hepato-pancreatic common duct near to the duodenum.

Due to the shortage of organ donors, the potential of hBTSCs to differentiate into hepatocytes or functional $\beta$ cells makes them an ideal source for regenerative medicine of midgut endodermal derived organs, including liver and pancreas [15-17]. Since portions of the extrahepatic biliary tree (e.g., cystic ducts, periampullary region) are easily available from donor livers and donor pancreata as well as from surgical resections, a new source of multipotent stem cells is available to be investigated for regenerative medicine of liver and pancreas. We also assume that these niches are the sources of cells for tissue turnover as well as sites vulnerable to oncogenic transformation [4-6]. Further details emerging from the careful phenotypic trait analyses of PBG cells, within the wall of bile ducts, support the interpretation of maturational lineages. Indeed, the biliary tree stem cells deep within the bile duct walls, near fibromuscular layers, co-express endodermal transcription factors essential for liver and pancreas formation (e.g., SOX9, SOX17, and PDX1) [4] (Figure 2). Moreover, they express pluripotency genes
(NANOG, OCT4, SOX2, KLF4, BMi-1, and Trop-2), stem cell surface markers (CD133, CXCR4, and CD44), and markers of self-replication (SALL4) and proliferation (e.g., Ki67), whereas markers of mature cells are negative including insulin and albumin [4]. PBGs closer to the bile duct lumens (but not at the luminal surface) have stem cells that still express many of these endodermal transcription factors, pluripotency genes, and stem cell surface markers but they now also express LGR5 (leucine-rich repeat-containing G protein coupled receptor 5) and EpCAM (epithelial cell adhesion molecule); the in situ studies have been clarified by findings from other studies (e.g., findings from cultures) to indicate that LGR5 and EpCAM are intermediate markers in the maturational process. Subsequent stages involve retention of key endodermal transcription factors (e.g., PDX1 or SOX17, but not both) in the nucleus (Figure 2). In the proximity of bile duct lumens and also in proximity of liver parenchyma or pancreas, the expression of pluripotency genes fades away; other stem cell traits are progressively lost (e.g., LGR5 or CD133 or SALL4); and mature markers appear and increase (e.g., albumin or insulin). These observations from our group 
have been recently confirmed by a lineage tracing study by Huch et al. [19], where LGR5 expression on hepatic stem cells has been confirmed as a marker identifying cells capable of reconstituting liver when transplanted into hosts.

\section{PBGs and Cholangiocarcinoma}

Cholangiocarcinoma (CCA) is the second most frequent primitive liver malignancy representing approximately 7$10 \%$ of the total liver malignancies. CCA may arise in any portion of the biliary tree. Currently, CCA is divided into intrahepatic (IH-CCA) and extrahepatic (EH-CCA), the latter comprising the perihilar and distal forms [20-27]. Gallbladder cancer and ampullar cancers are not considered part of the CCA classification. The IH-CCA may emerge from the smallest portion of the intrahepatic biliary tree up to the second order bile ducts, while, as far as the EH-CCA is concerned, the separation point between perihilar (Klatskin tumour) and distal EH-CCA is located at the insertion of the cystic duct (Figure 3), according to WHO classification [28]. CCA is a very heterogeneous cancer, from any point of view, including its epidemiology, risk factors, morphology, pathology, molecular pathology, modalities of growth, and clinical features [27, 29-36]. The heterogeneity involves even more of the IH- and EH- forms that are two completely different cancers from any point of view. Despite the histologic heterogeneity, recent studies have demonstrated $\mathrm{IH}-$ CCA is represented essentially by two main forms [37]. One form, called mucin-CCA, is entirely constituted by mucinsecreting adenocarcinomas and, from a pathologic point of view, is indistinguishable from the EH-CCA. A second form, called mixed-CCA, is peripherally located inside the liver and is composed of neoplastic ductular proliferation consisting of small monotonous and/or anastomosing glands, strongly positive for $\mathrm{K} 7$ and K19, with tumor boundary characterized by HCC-like trabecular area and, in some cases, small areas of adenocarcinoma with scarce mucin production [37-40]. The mixed type of IH-CCA shares common features with cholangiolocellular carcinoma (CLC), almost entirely constituted by neoplastic ductular proliferation, and with CK19+ hepatocellular carcinoma [37-42]. Taking into consideration the morphology, immunohistochemistry, and molecular analyses, the mixed-CCA likely originates from cholangiocytes located in the interlobular bile ducts and stem/progenitor located in the canals of Hering, while the mucin-CCA from mucin secreting epithelial cells lining large intrahepatic or extrahepatic ducts. Importantly, this cell-of-origin based distinction between mucin- and mixedCCA reflects completely different clinical, pathological, and radiological findings. Thus, the cell-of-origin based CCA classification appears to be more clinically relevant than the current anatomical based CCA classification [27, 29-31, 38, 42] (Figure 3). The emerging distinctions between mixed and pure mucin IH-CCA reflects the heterogeneity of biliary epithelium lining bile ducts of different size. Our recent findings on PBGs shed a new light on this heterogeneity. Indeed, the two stem cells compartments, the canals of Hering, and PBGs, which sustain the development of separate lineages within the hepatic parenchyma, indicate separate candidate cells of origin for CCA subtypes [29]. Indeed, a number of recent observations suggest that mucin- and mixed-CCA may arise from these different stem cell compartments in the liver [30, 40-42]. In detail, the first type of stem cell niche is located in the canal of Hering and is primarily comprised of bipotential stem cells (hHpSCs) and their descendants, hepatoblasts (hHBs), and both are able to give rise to lineages of hepatocytes and cholangiocytes $[43,44]$ (Figure 3). Analyses of hHpSCs and hHBs suggest that, under known culture conditions, the hHpSCs, but not the hHBs, are able to self-replicate; consequently, we hypothesize that the hHBs are the liver's equivalent of transit amplifying cells that in many tissues are the acute responders to various regenerative demands. Apart from mixed-CCA, liver cancers originating from $\mathrm{hHpSC}$-derived lineages also comprise the combined hepatocellular-cholangiocarcinoma (HCC-CCA) and CLC [30, 40-42, 45]. Pathologic differences between these different cancers depend on the lineage stage that is malignantly transformed; the phenotypic traits of the cells are lineage dependent changing stepwise with the differentiation of the cell of origin towards mature hepatocytes or cholangiocytes.

A second stem cell niche is located at the bottom of PBGs $[2,4,5]$ (Figure 3 ) and is composed of multipotent endodermal stem/progenitor cells (hBTSCs). Similarities exist between PBGs, pancreatic ductal glands (PDGs), and colon crypts in terms of cell components and stem/progenitors cells and this is justified by the common embryologic origins. Therefore, at the bottom of these three types of glands, similar niches of stem/progenitor cells exist as remnants of fetal life. The similarities of these niches may explain the existence of pathologies contemporary involving these organs (ulcerative colitis (UC), primary sclerosing cholangitis (PSC), and autoimmune pancreatitis) or the similarities between cancers involving pancreas, the biliary tree, and the colon. There are a number of recent observations suggesting that PBGs are involved in the emergence of mucin-CCAs including the following: (1) the density of PBGs in the biliary tree is the highest in the typical sites of CCA emergency that include the hilus, the branching points, and periampullary region; (2) PBGs that are activated undergo hyperplasia and mucinous metaplasia in pathologies at risk for CCA development, including PSC [46]; (3) cancer stem cells (CSCs) identified in human mucin-CCAs and expressing markers, such as LGR5 [47], on stem/progenitor cells exclusively located at the bottoms of PBGs $[2,4,5]$. Therefore, it is becoming evident that mucin-CCA is a cancer of glandular origin, and this could explain the similarities with ductal adenocarcinoma of the pancreas and colorectal cancer. Indeed, similar to mucin-CCA, pancreatic cancer may arise in the context of an intraductal papillary mucinous cystic neoplasm (IPMN), a lesion originating in the peripancreatic glands within the pancreatic ducts, the counterpart of the IPNB in bile ducts $[45,48-51]$. As far as intestine is concerned, the involvement of colon crypts in the origin of colorectal cancer is largely documented. PBGs undergo hyperplasia and mucinous metaplasia in chronic inflammatory pathologies of the biliary tree including PSC, IgG4-associated sclerosing cholangitis, and intrahepatic lithiasis $[46,52]$. In PSC the degree of PBG 
involvement in terms of proliferation and inflammation correlates with the degree of inflammation in the bile duct wall. The same occurs for pancreatic duct glands that are activated, proliferate, and undergo metaplastic changes in pathologies at risk for pancreatic cancer such us diabetes and pancreatitis [51]. Mucin-CCA, ductal pancreatic adenocarcinoma, and colorectal cancer could have origins from a common stem cell located at the bottom of PBGs, PDGs, and colon crypts, respectively. Many efforts by researchers worldwide have been focused on the candidate cells of origin of cancer. The general opinion is that the cell of origin of different cancers possesses properties of a stem/progenitor cell [45]. As far as CCA is concerned, this topic has been scarcely explored, and this is also because of the rarity of this cancer and its biological heterogeneity. However, the recent discovery of different stem cell compartments in the human biliary tree represents a consistent advance also for understanding CCA origins. Epithelial cell-adhesion molecule (EpCAM), expressed by the intermediate cell compartment in biliary tree stem cell niches, has been considered recently as a marker of the CCA cell of origin [47, 53-59] (Figure 2). EpCAM/Tacstd is a single transmembrane glycoprotein originally identified as a marker of epithelial-derived carcinomas. It was proposed to be a homophilic cell-cell adhesion molecule in early studies $[53,54]$ and has recently been implicated in proliferation, differentiation, cell migration, and metastasis [55-57]. A recent paper by Lu et al. illustrated how an EpCAM-dependent mechanism confers to endodermal cells the competence to respond to the liver-inductive Wnt2bb signal [58]. These results demonstrated that EpCAM directly binds to Kremen1, disrupting the interaction between Dickkopf2 (Dkk2) and Kremenl, thereby stabilizing the low-density lipoprotein receptor-related protein 6 (Lrp6) on the cell surface. This allows formation of active Lrp6-signalosomes and thus enables derepressing and cooperatively activating Wnt signaling stimulated by mesenchymal-endodermal interactions. Notably, EpCAM positive hHpSCs in adult life are driven by macrophage derived Wnt to specify hepatic progenitor cell fate in chronic liver diseases [56]. Moreover, Wnt $/ \beta$ catenin signaling has been involved in liver carcinogenesis since it enhances hypoxia-induced epithelial-mesenchymal transitions. Thus, a cross-talk between EpCAM+ stem cells and mesenchyma could sustain a Wnt-induced carcinogenesis in the diseased liver. As far as hBTSCs are concerned, EpCAM expression is acquired after that of LGR5 in the intermediate stages of the differentiative pathways and is lost upon completion of biliary differentiation [4]. The differentiative arrest that characterizes the carcinogenetic process could follow the expansion and proliferation of these intermediate, EpCAM+ biliary stem/progenitor cell subpopulations. In agreement with this hypothesis, EpCAM expression has a strong negative prognostic impact in human CCA (unspecified morphology), in HCCs expressing stem cell features (CK19+ HCC), and in combined HCC-CCA [24, $41,59]$. We have recently observed [47] that mucin-positive established CCA cell lines are almost entirely constituted by EpCAM+ cells which are virtually absent in mucin-negative CCA cell lines. This further supports the concept that mucinand mixed human CCA display different cells of origin and that an EpCAM+ cell could represent a candidate cell for mucin-CCA. Going back to the similarities between CCA, ductal pancreatic adenocarcinoma, and colorectal cancer, EpCAM overexpression has been correlated with malignant potential of IPMNs [60] and a novel genetic disorder of Lynch syndrome characterized by EpCAM gene deletion has been recently described [61]. These observations further support the hypothesis of a common cell of origin of these cancers $(\mathrm{EpCAM}+$ cell) located in the glands of the original tissues (Figure 2). Lgr5 (leucine-rich repeat-containing G protein coupled receptor 5) is another candidate stem cell marker that could be involved in CCA pathogenesis [19, 62-64]. Lgr5 is a Wnt target gene that marks actively dividing stem cells in Wnt-driven, self-renewing tissues such as small intestine, colon, stomach, and hair follicles [19]. In our studies, Lgr5 also marks a subset of the stem/progenitors located in the bottom of PBGs $[2,4,5]$. Lgr5 is a functional intestinal stem cell marker and a selective marker for human colorectal CSCs [62]. Recently a subclass of the Lgr5 positive intestinal stem cell compartment has been identified as a potential specific CSC in colon neoplasia [62]. These Lgr5+ CSCs expressed specifically doublecortin-like kinase 1 (Dclk1) and continuously produced tumor progeny in the polyps of ApcMin/+ mice. Specific ablation of Dclk1-positive CSCs resulted in a marked regression of polyps without apparent damage to the normal intestine. Thus Lgr5/DclK1 + cells represent a potential target in endodermal derived cancers [62].

Using a lineage tracing method, it has been demonstrated recently that Lgr5-lacZ is not expressed in healthy adult liver. However, small cells that are Lgr5-LacZ1 positive appear near bile ducts upon damage, coinciding with robust activation of Wnt signaling [19]. A role of Lgr5+ cells as CSC has been recently suggested in undifferentiated liver cancers [63, 64]. We have recently described [47] how Lgr5 positivity predominated in mucin-CCA with respect to mixed-CCA. This evidence supports our previous hypothesis, based on clinical, pathological, and epidemiological observations, that pure mucin-CCA represents a cancer originating from PBGs, making this cancer very similar biologically to colon cancer; Lgr5+ cells in glandular crypts have been considered to play a carcinogenetic role in such cancers. Consistent with the origin of mucin-CCA from PBGs, Sato et al. recently described a privileged spreading of hilar CCA via the PBG network [65].

The discovery of hBTSCs within PBGs also leads to the recent observation that the biliary tree-derived stem cells and their connections to pancreatic committed progenitors constitute a biological framework for life-long pancreatic organogenesis [2]. On a pathophysiologic level, this connection is sustained by much evidence, which finally leads to the existence of common stem/progenitor cells for pancreas and biliary tree from fetal life and persisting into adulthood. PDGs have been described recently as a new compartment of the major pancreatic duct in humans and mice [51]. As for PBGs, the cells of the PDGs are sites of increased vulnerability to diseases, especially in response to the injury of mature cells or to chronic inflammatory stimuli which represent the initial step of the oncogenic process [51, 65]. In this 
regard, it is noteworthy that sonic hedgehog (Shh), a pivotal signaling pathway involved in biliopancreatic development, is also involved in pathologies of biliary tree and pancreatic ducts where it sustains cell proliferation. In particular, it is emerging that Shh-mediated mucin-metaplasia of PDGs and/or of PBGs is associated with chronic injury [66]. Thus, emerging evidence supports similarities between biliary and pancreatic carcinogenesis in terms of both cells of origin and underlying molecular pathways. In the same direction, pathologies considered risk factors for mucin-CCA and pancreatic cancer are characterized by the activation of the respective stem cell compartment in PBGs or PDGs $[27,29]$. This includes diabetes and chronic pancreatitis for pancreatic cancer and PSC for mucin-CCA $[27,29,66]$. PSC is frequently associated with chronic pancreatitis where both PBG and PDG cells proliferate as a consequence of chronic inflammation $[27,29,51,66]$. Diabetes and insulin resistance (obesity) are recognized risk factors for CCA and pancreatic adenocarcinoma [27, 29]. How diabetes (and insulin resistance) predisposes to such cancers remained a speculation for years. However, in obese and type II diabetic mice, proliferation of stem/progenitor cells within PDGs has been observed in association with the appearance of distinct tumor markers (Muc5ac, S100P, regenerating islet derived 3b, 14-3-3 s, and prostate stem cell antigen) [66]. Collectively, the evidence concerning involvement of PBG and PDG in pathologies at risk for cancer development suggests that CCA and pancreatic tumors might originate from PBGs or PDGs, respectively.

Similarities between CCA, pancreatic adenocarcinoma, and colorectal cancer also concern molecular pathways underlying oncogenesis. For example, a pivotal carcinogenetic pathway involves K-RAS mutations largely documented in colorectal and pancreatic cancers. Ras proteins (Kras, Nras, Hras, and Braf), responsible for signal transduction downstream to growth factor receptors, have been investigated largely in CCA. In this regard, KRAS-activating mutations represent one of the most frequent genetic alterations (up to $75 \%$ of CCA cases) [67]. When classified by tumor site, only $17 \%$ of peripheral-type CCAs (mixed-CCAs) were positive for KRAS mutations, whereas this occurs in more than $50 \%$ of the hilar-type CCA, that is, typically a mucin-CCA [67]. Thus, also molecular profiles underlie the similarities between mucin-producing cancers of the colon, pancreas, and biliary tree. The specific glands within the tissues (intestinal crypts, PDGs, and PBGs) contain stem/progenitor cells which are candidate cancer cells of origin, share common morphological and phenotype features, and could represent a major determinant of the molecular and biological similarities of these adenocarcinomas.

In substance, evidence is emerging that PBGs could be involved in the pathogenesis of mucin-CCA. It could be argued that biliary dysplasia, the most common premalignant condition of large-duct CCA [20], usually occurs in the bile duct surface epithelium. However, PBGs play a role in the renewal of surface epithelium $[2,4,5]$ and in most CCA risk factors (PSC, liver flukes, and hepatolithiasis) damage of biliary epithelium reasonably leads to its enhanced turnover, as indicated by enhanced proliferation of surface epithelium and PBGs $[46,68,69]$. Therefore, a possible involvement of PBGs also in biliary dysplasia should merit extensive research.

\section{PBG Are Activated in Pathologies at Risk of CCA}

A number of risk factors have been described for CCA with large racial and geographic differences [33]. The more robust risk factor for CCA in Western countries is PSC, while liver flukes and intrahepatic lithiasis are risk factors in the eastern world [33]. PSC is a disease affecting both intrahepatic and extrahepatic bile ducts and, indeed, it represents the strongest independent risk factor for both IH-CCA and EH-CCA with a cumulative risk of $1-5 \%$ at 10 years [27, 33]. Inflammatory bowel diseases possibly associated with PSC may further increase CCA risk, cumulative risk of $10-15 \%$ at 10 years $[27,33]$. Recent studies indicate how PBGs are activated in the course of PSC and IgG4-related PSC $[46,52]$. Specifically, Terasaki et al. examined the histological changes of the PBGs in seven PSC patients and showed how these glands display proliferation, nonspecific inflammation with lymphoplasmacytic infiltration, fibrosis, and destruction [46]. In addition, there were cystic lesions around the bile ducts, and they were considered to reflect dilatation of the PBGs. These changes were found around the intrahepatic and extrahepatic bile ducts. It is of interest that changes in the PBGs tended to correlate with the inflammatory changes of the bile ducts. These findings suggest that the PBGs are targets of damage in PSC. Stem cell niches with the PBGs recapitulate the intestinal model, in the sense that stem cell niches reside deep within the glandular elements (Lieberkuhn's crypts in the intestine), and differentiate toward distinct fates, maturing in an upward direction (crypt-villus axis in the intestine, radial axis in bile ducts) $[2,4]$. The development of PSC and UC appears to precede dysplasia of the biliary and colonic mucosa, respectively, and represents a marker of patients at risk of cancer [27]. The common embryologic origins of biliary tree and intestine and the similarities in the stem cell niches residing in the two organs lead to the suggestion that the inflammatory immune-mediated processes underlining PSC and UC could target cells originating from biliary and intestinal lineages similarly and indistinguishably. This opens new perspectives on current knowledge of these pathologies.

The evidence for a possible association between liver flukes and CCA has been evaluated by the International Agency for Research on Cancer (IARC) since 1994 [70]. Opisthorchis viverrini and Clonorchis sinensis infections inhabit mainly in the IH and EHBDs and, more rarely, in the gallbladder and pancreatic ducts. The infection is associated with a number of hepatobiliary diseases, including cholangitis, cholecystitis, and hepatolithiasis and with several pathological changes in the liver, gallbladder, and EHBDs [71-73]. Microscopically, the intrahepatic lesions are confined to the biliary tree, particularly to the large and medium-sized bile ducts where the flukes are harboured [70-75]. During liver fluke infection, inflammation, periductal fibrosis, and hyperplastic and dysplastic responses of PBGs and surface bile duct epithelium may represent predisposing lesions that 
enhance the susceptibility of DNA to carcinogens including several $N$-nitroso compounds and their precursors [74, 75]. Liver fluke-associated CCAs display distinct features with respect to sporadic CCAs [68]. Indeed, it has been observed that live fluke-associated CCAs display intestinal goblet cell phenotype and p53 overexpression much more frequently than sporadic CCAs, indicating differences in the oncogenic processes [68].

\section{Conclusions}

Stem cells and progenitors in PBGs within the biliary tree provide functions similar to that of stem cells and progenitors in intestinal crypts and of committed progenitors found in PDGs within pancreatic ducts. These glandular structures, containing stem cells and/or committed progenitor cells of endodermal origin, are all involved in the renewal of surface epithelium and are the target of damage in inflammatory processes or neoplastic diseases of the respective tissues. As far as the biliary tree is concerned, many different observations are highlighting a relevant role of PBGs in the pathogenesis of PSC and CCA. Accumulating evidence, in fact, suggests that the pure mucin-CCA, emerging from large sized intrahepatic or extrahepatic bile ducts, is a cancer originating from the hBTSCs located in the PBGs. This evidence could explain the molecular and biological similarities between mucin-CCA, pancreatic ductal adenocarcinomas originating from stem cells within PBGs of the hepato-pancreatic common duct near the duodenum or from committed progenitors within PDGs, and colorectal cancers originating from stem/progenitors within colon crypts. In this regard, ongoing research investigations are investigating possible CSCs that are shared in common by these three types of cancers. Findings regarding this should have enormous clinical and therapeutic implications.

\section{Abbreviations}

hBTSCs: Human biliary tree stem/progenitor cells

CCA: Cholangiocarcinoma

CLC: Cholangiolocarcinoma

CD133: Prominin 1

CK: $\quad$ Cytokeratin

CSCs: Cancer stem cells

CXCR4: CXC-chemokine receptor 4

Dclk1: Doublecortin-like kinase 1

EpCAM: Epithelial cell adhesion molecule

EH: $\quad$ Extrahepatic

EH-CCA: Extrahepatic cholangiocarcinoma

EHBDs: Extrahepatic bile ducts

HCC: Hepatocellular carcinoma

hHpSCs: Human hepatic stem/progenitor cells

IH: Intrahepatic

IH-CCA: Intrahepatic cholangiocarcinoma

IHBDs: Intrahepatic bile ducts

IPMN: Intraductal papillary mucinous cystic neoplasm (a lesion originating in the pancreatic ductal glands)
IPNB: Intraductal papillary neoplasm of the bile duct

LGR5: Leucine-rich repeat-containing G protein coupled receptor 5

MUC: $\quad$ Mucin

NANOG: Nanog homeobox

OCT4: $\quad$ POU class 5 homeobox 1

PBGs: Peribiliary glands

PDGs: Pancreatic duct glands

PDX1: Pancreatic and duodenal homeobox 1

PSC: $\quad$ Primary sclerosing cholangitis

SALL4: Sal-like protein 4

SHH: Sonic hedgehog

SOX: $\quad$ Sry-related HMG box

UC: $\quad$ Ulcerative colitis.

\section{Conflict of Interests}

The authors declare that there is no conflict of interests regarding the publication of this paper.

\section{Acknowledgments}

Domenico Alvaro was supported by FIRB Grant no. RBAP10Z7FS_004 and by PRIN Grant no. 2009X84L84_002. V. Cardinale was supported by FIRB Grant no. RBAP10Z7FS 004. E. Gaudio was supported by research project grant from the University "Sapienza" of Rome and FIRB Grant no. RBAP10Z7FS_001 and PRIN Grant no. 2009X84L84_001. The study was also supported by Consorzio Interuniversitario Trapianti d'Organo, Rome, Italy, and by a sponsored research agreement (SRAs) from Vesta Therapeutics (Bethesda, MD). LM Reid is funded also by an SRA from Vesta Therapeutics (Bethesda, MD), by an NCI Grant (RCA-182322A), and by an NCI Center Grant (CA016086).

\section{References}

[1] S. G. Royce, N. R. Hughes, S. Binos, J. R. Underwood, and P. S. Bhathal, "Vertebrate phylogeny of antigen D10: identification of a conserved foregut cell lineage," Histochemistry and Cell Biology, vol. 114, no. 2, pp. 125-135, 2000.

[2] V. Cardinale, Y. Wang, G. Carpino et al., "The biliary tree-a reservoir of multipotent stem cells," Nature Reviews Gastroenterology and Hepatology, vol. 9, no. 4, pp. 231-240, 2012.

[3] Y. Nakanuma, M. T. Hoso, T. Sanzen et al., "Microstructure and development of the normal and pathologic biliary tract in humans, including blood supply," Microscopy Research and Technique, vol. 38, pp. 552-570, 1997.

[4] G. Carpino, V. Cardinale, P. Onori et al., "Biliary tree stem/progenitor cells in glands of extrahepatic and intraheptic bile ducts: an anatomical in situ study yielding evidence of maturational lineages," Journal of Anatomy, vol. 220, no. 2, pp. 186-199, 2012.

[5] V. Cardinale, Y. Wang, G. Carpino et al., "Multipotent stem/progenitor cells in human biliary tree give rise to hepatocytes, cholangiocytes, and pancreatic islets," Hepatology, vol. 54, no. 6, pp. 2159-2172, 2011.

[6] R. Semeraro, G. Carpino, V. Cardinale et al., "Multipotent stem/progenitor cells in the human fetal bilary tree," Journal of Hepatology, vol. 57, pp. 987-994, 2012. 
[7] T. Terada, T. Morita, M. Hoso, and Y. Nakanuma, "Pancreatic enzymes in the epithelium of intrahepatic large bile ducts and in hepatic bile in patients with extrahepatic bile duct obstruction," Journal of Clinical Pathology, vol. 47, no. 10, pp. 924-927, 1994.

[8] J. H. Youson and A. A. Al-Mahrouki, "Ontogenetic and phylogenetic development of the endocrine pancreas (islet organ) in fishes," General and Comparative Endocrinology, vol. 116, no. 3, pp. 303-335, 1999.

[9] T. Terada and Y. Nakanuma, "Pancreatic lipase is a useful phenotypic marker of intrahepatic large and septal bile ducts, peribiliary glands, and their malignant counterparts," Modern Pathology, vol. 6, no. 4, pp. 419-426, 1993.

[10] T. Terada and Y. Nakanuma, "Expression of pancreatic enzymes $(\alpha$-amylase, trypsinogen, and lipase) during human liver development and maturation," Gastroenterology, vol. 108, no. 4, pp. 1236-1245, 1995.

[11] T. A. Roskams, N. D. Theise, C. Balabaud et al., "Nomenclature of the finer branches of the biliary tree: canals, ductules, and ductular reactions in human livers," Hepatology, vol. 39, no. 6, pp. 1739-1745, 2004.

[12] C. E. L. Tan and G. J. Moscoso, “The developing human biliary system at the porta hepatis level between 11 and 25 weeks of gestation: a way to understanding biliary atresia. Part 2," Pathology International, vol. 44, no. 8, pp. 600-610, 1994.

[13] T. Terada and Y. Nakanuma, "Development of human intrahepatic peribiliary glands: histological, keratin immunohistochemical, and mucus histochemical analyses," Laboratory Investigation, vol. 68, no. 3, pp. 261-269, 1993.

[14] S. Y. S. Si Young Song, M. Gannon, M. K. Washington et al., "Expansion of Pdxl-expressing pancreatic epithelium and islet neogenesis in transgenic mice overexpressing transforming growth factor $\alpha$," Gastroenterology, vol. 117, no. 6, pp. 1416-1426, 1999.

[15] S. Bonner-Weir and G. C. Weir, "New sources of pancreatic $\beta$ cells," Nature Biotechnology, vol. 23, no. 7, pp. 857-861, 2005.

[16] M. Borowiak and D. A. Melton, "How to make $\beta$ cells?" Current Opinion in Cell Biology, vol. 21, no. 6, pp. 727-732, 2009.

[17] E. Kroon, L. A. Martinson, K. Kadoya et al., "Pancreatic endoderm derived from human embryonic stem cells generates glucose-responsive insulin-secreting cells in vivo," Nature Biotechnology, vol. 26, no. 4, pp. 443-452, 2008.

[18] Y. Wang, G. Lanzoni, G. Carpino et al., "Biliary tree stem cells are precursors to pancreatic committed progenitors: evidence for possible pancreatic organogenesis throughout life," Stem Cells, vol. 31, pp. 1966-1979, 2013.

[19] M. Huch, C. Dorrell, S. F. Boj et al., "In vitro expansion of single $\mathrm{Lgr}^{+}$liver stem cells induced by Wnt-driven regeneration," Nature, vol. 494, pp. 247-250, 2013.

[20] F. Callea, C. Sergi, G. Fabbretii, M. Brisigoiti, C. Cozzutto, and D. Medicina, "Precancerous lesions of the biliary tree," Journal of Surgical Oncology, vol. 53, no. 3, pp. 131-133, 1993.

[21] Y. Nakanuma, H. Minato, T. Kida et al., "Pathology of cholangiocellular carcinoma," in Primary Liver Cancer in Japan, T. Tobe, H. Kameda, M. Okudaira, and M. Ohto, Eds., pp. 39-50, Springer, Tokyo, Japan, 1994.

[22] G. Klatskin, "Adenocarcinoma of the hepatic duct at its bifurcation within the porta hepatis. An unusual tumor with distinctive clinical and pathological features," The American Journal of Medicine, vol. 38, no. 2, pp. 241-256, 1965.

[23] Y. Nakanuma, A. S. Leong -Y, B. Sripa et al., "Intra-hepatic cholangiocarcinoma," in Pathology and Genetics of Tumours of the Digestive, S. R. Hamilton and L. A. Aaltonen, Eds., World Health Organization Classification of Tumours. Lyon IARC Press, 2000.

[24] T. Roskams, "Liver stem cells and their implication in hepatocellular and cholangiocarcinoma," Oncogene, vol. 25, no. 27, pp. 3818-3822, 2006.

[25] T. M. Welzel, K. A. McGlynn, A. W. Hsing, T. R. O’Brien, and R. M. Pfeiffer, "Impact of classification of hilar cholangiocarcinomas (Klatskin Tumors) on the incidence of intra- and extrahepatic cholangiocarcinoma in the United States," Journal of the National Cancer Institute, vol. 98, no. 12, pp. 873-875, 2006.

[26] D. Alvaro, E. Crocetti, S. Ferretti, M. C. Bragazzi, and R. Capocaccia, "Descriptive epidemiology of cholangiocarcinoma in Italy," Digestive and Liver Disease, vol. 42, no. 7, pp. 490-495, 2010.

[27] V. Cardinale, R. Semeraro, A. Torrice et al., "Intra-hepatic and extra-hepatic cholangiocarcinoma: new insight into epidemiology and risk factors," World Journal of Gastrointestinal Oncology, vol. 2, pp. 407-416, 2010.

[28] N. D. Theise, O. Nakashima, Y. N. Park et al., WHO Classification of Tumours of the Digestive System, Lyon IARC Press, 2010.

[29] V. Cardinale, G. Carpino, L. Reid et al., "Multiple cells of origin in choalngiocarcinoma underlie biological, epidemiological and clinical heterogenity," World Journal of Gastrointestinal Oncology, vol. 4, pp. 94-102, 2012.

[30] V. Cardinale, G. Carpino, L. M. Reid et al., "Cholangiocarcioma a cancer in search of the right classification," Hepatology, vol. 56, pp. 1585-1596, 2012.

[31] V. Cardinale, Y. Wang, G. Carpino et al., "Mucin-producing cholangiocarcinoma might derive from biliary tree stem/progenitor cells located in peribiliary glands," Hepatology, vol. 55, no. 6, pp. 2041-2042, 2012.

[32] M. Gatto and D. Alvaro, "New insights on cholangiocarcinoma," World Journal of Gastrointestinal Oncology, vol. 2, no. 3, pp. 136145, 2010.

[33] M. C. Bragazzi, V. Cardinale, G. Carpino et al., "Cholangiocarcinoma: epidemiology and risk factors," Translational Gastrointestinal Cancer, vol. 1, no. 1, pp. 21-32, 2012.

[34] D. Alvaro, M. C. Bragazzi, A. Benedetti et al., "Cholangiocarcinoma in Italy: a national survey on clinical characteristics, diagnostic modalities and treatment. Results from the "Cholangiocarcinoma" committee of the Italian Association for the Study of Liver disease," Digestive and Liver Disease, vol. 43, no. 1, pp. 60-65, 2011.

[35] M. Gatto, M. C. Bragazzi, R. Semeraro et al., "Cholangioacrcinoma: update and future perspectives," Digestive and Liver Disease, vol. 42, pp. 253-260, 2010.

[36] D. Alvaro, E. Crocetti, S. Ferretti et al., "Descriptive epidemiology of chialngiocarcinoma in Italy," Digestive and Liver Disease, vol. 42, no. 7, pp. 490-495, 2010.

[37] M. Komuta, O. Govaere, V. Vandecaveye et al., "Histological diversity in cholangiocellular carcinoma reflects the different cholangiocyte phenotypes," Hepatology, vol. 55, no. 6, pp. 1876$1888,2012$.

[38] V. Cardinale, G. Carpino, Y. Wang et al., "Biliary tree stem/progenitor cells and perspectives in physiopathology and regenerative medicine," Translational Gastrointestinal Cancer, vol. 2, pp. 30-38, 2013.

[39] G. Carpino, V. Cardinale, L. Reid et al., "Cells of origin and cancer stem cells in cholangiocarcinoma," Translational Gastrointestinal Cancer, vol. 1, pp. 33-43, 2012. 
[40] M. Komuta, B. Spee, S. V. Borght et al., "Clinicopathological study on cholangiolocellular carcinoma suggesting hepatic progenitor cell origin," Hepatology, vol. 47, no. 5, pp. 1544-1556, 2008.

[41] J.-S. Lee, J. Heo, L. Libbrecht et al., "A novel prognostic subtype of human hepatocellular carcinoma derived from hepatic progenitor cells," Nature Medicine, vol. 12, no. 4, pp. 410-416, 2006.

[42] V. Cardinale, M. C. Bragazzi, G. Carpino et al., "Cholangiocarcinoma: increasing burden of classifications," Hepatobiliary Surgery and Nutrition, vol. 2, pp. 272-280, 2013.

[43] E. Schmelzer, L. Zhang, A. Bruce et al., "Human hepatic stem cells from fetal and postnatal donors," Journal of Experimental Medicine, vol. 204, no. 8, pp. 1973-1987, 2007.

[44] R. Turner, O. Lozoya, Y. Wang et al., "Human hepatic stem cell and maturational liver lineage biology," Hepatology, vol. 53, no. 3, pp. 1035-1045, 2011.

[45] V. Cardinale, G. Carpino, L. M. Reid et al., "Notch2 signaling and undifferentiated liver cancers: evidence of hepatic stem/ progenitor cell origin," Hepatology, vol. 58, no. 3, article 1188, 2013.

[46] S. Terasaki, Y. Nakanuma, M. Unoura, S. Kaneko, and K. Kobayashi, "Involvement of peribiliary glands in primary sclerosing cholangitis: a histopathologic study," Internal Medicine, vol. 36, no. 11, pp. 766-770, 1997.

[47] A. Torrice, G. Carpino, A. Fraveto et al., "Characterization of cancer stem cells in human cholangiocarcinoma (CCA) subtypes," Hepatology, abstract 1086A, 2013.

[48] Y. Nakanuma and Y. Sato, "Cystic and papillary neoplasm involving peribiliary glands: a biliary counterpart of branchtype IPMN?" Hepatology, vol. 55, pp. 2040-2041, 2012.

[49] F. G. Rocha, H. Lee, N. Katabi et al., "Intraductal papillary neoplasm of the bileduct: a biliry equivalent to intraductal papillary mucinous neoplasm of the pancreas?" Hepatology, vol. 56, no. 4, pp. 1352-1360, 2012.

[50] M. Massani, T. Stecca, L. Fabris et al., "Isolation and characterization of biliary epithelial and stromal cells from resecate human cholangiocarcinoma: a novel in vitro model to study tumor-stroma interactions," Oncology Reports, vol. 30, pp. 11431148, 2013.

[51] O. Strobel, D. E. Rosow, E. Y. Rakhlin et al., "Pancreatic duct glands are distinct ductal compartments that react to chronic injury and mediate Shh-induced metaplasia," Gastroenterology, vol. 138, no. 3, pp. 1166-1177, 2010.

[52] Y. Nakanuma and Y. Zen, "Pathology and immunopathology of immunoglobulin G4-related sclerosing cholangitis: the latest addition to the sclerosing cholangitis family," Hepatology Research, vol. 37, no. 3, pp. S478-S486, 2007.

[53] S. V. Litvinov, M. P. Velders, H. A. M. Bakker, G. J. Fleuren, and S. O. Warnaar, "Ep-CAM: a human epithelial antigen is a homophilic cell-cell adhesion molecule," Journal of Cell Biology, vol. 125, no. 2, pp. 437-446, 1994.

[54] S. V. Litvinov, M. Balzar, M. J. Winter et al., "Epithelial cell adhesion molecule (Ep-CAM) modulates cell-cell interactions mediated by classic cadherins," Journal of Cell Biology, vol. 139, no. 5, pp. 1337-1348, 1997.

[55] M. Trzpis, P. M. J. McLaughlin, L. M. F. H. De Leij, and M. C. Harmsen, "Epithelial cell adhesion molecule: more than a carcinoma marker and adhesion molecule," The American Journal of Pathology, vol. 171, no. 2, pp. 386-395, 2007.

[56] L. Boulter, O. Govaere, T. G. Bird et al., "Macrophage-derived Wnt opposes Notch signaling to specify hepatic progenitor cell fate in chronic liver disease," Nature Medicine, vol. 18, no. 4, pp. 572-579, 2012.

[57] Q. Zhang, X. Bai, W. Chen et al., "Wnt/ $\beta$-catenin signaling enhances hypoxia-induced epithelial-mesenchymal transition in hepatocellular carcinoma via crosstalk with hif-1 $\alpha$ signaling," Carcinogenesis, vol. 34, pp. 962-973, 2013.

[58] H. Lu, J. Ma, Y. Yang et al., "EpCAM is an endoderm-specific Wnt derepressor that licenses hepatic development," Developmental Cell, vol. 24, no. 5, pp. 543-553, 2013.

[59] T. Yamashita, J. Ji, A. Budhu et al., "EpCAM-positive hepatocellular carcinoma cells are tumor-initiating cells with stem/progenitor cell features,' Gastroenterology, vol. 136, no. 3, pp. 1012.e4-1024.e4, 2009.

[60] S. Yonaiyama, Y. Toyoki, S. Morohashi et al., "Ephitelial cell adhesion molecule (EpCAM) overexpression is correlated with malignant potentials of intraductal papillary mucinous neoplasms (IPMNs) of the pancreas," BioMed Research, vol. 34, no. 2, pp. 87-95, 2013.

[61] N. Tomita, T. Yamano, N. Matsubara et al., "A novel genetic disorder of Lynch syndrome-EpCAM gene deletion," Gan to Kagaku Ryoho, vol. 40, pp. 143-147, 2013.

[62] Y. Nakanishi, H. Seno, A. Fukuoka et al., "Dclk1 distinguishes between tumor and normal stem cells in the intestine," Nature Genetics, vol. 45, pp. 98-103, 2013.

[63] M. Fukuma, K. Tanese, K. Effendi et al., "Leucine-rich repeatcontaining G protein-copled receptor 5 regulates epithelial cell phenotype and survival of hepatocellular carcionoma cells," Experimental Cell Research, vol. 319, pp. 113-121, 2013.

[64] Y. Yamamoto, M. Sakamoto, G. Fujii et al., "Overexpression of orphan G-protein-coupled receptor, Gpr49, in human hepatocellular carcinomas with $\beta$-catenin mutations," Hepatology, vol. 37, no. 3, pp. 528-533, 2003.

[65] H. Sato, Y. Nakanuma, K. Kozaka et al., "Spread of hilar cholangiocarciomas via peribiliary gland network: a hither-tounrecognized route of periductal infiltration," International Journal of Clinical and Experimental Pathology, vol. 6, no. 2, pp. 318-322, 2013.

[66] A. Bobrowski, M. Spitzner, S. Bethge et al., "Risk factorsfor pancreatic ductal adenocarcinoma specifically stimulate pancreatic duct glands in mice," The American Journal of Pathology, vol. 182, no. 3, pp. 965-974, 2013.

[67] D. Alvaro and V. Cardinale, "Molecular profiling," in Biliary Tract and Gallbladder Cancer: A Multidisciplinary Approach, J. M. Herman, T. Pawlik, and C. R. Thomas Jr., Eds., Medical Radiology, pp. 99-117, 2nd edition, 2014, http://www.springer.com/ medicine/radiology/book/978-3-642-40557-0.

[68] N. R. Hughes, C. Pairojkul, S. G. Royce, A. Clouston, and P. S. Bhathal, "Liver fluke-associated and sporadic cholangiocarcinoma: an immunohistochemical study of bile duct, peribiliary gland and tumour cell phenotypes," Journal of Clinical Pathology, vol. 59, no. 10, pp. 1073-1078, 2006.

[69] W. M. S. Tsui, P. W. Y. Lam, W.-K. Lee, and Y.-K. Chan, "Primary hepatolithiasis, recurrent pyogenic cholangitis, and oriental cholangiohepatitis - a tale of 3 countries," Advances in Anatomic Pathology, vol. 18, no. 4, pp. 318-328, 2011.

[70] D. M. Parkin, "The global health burden of infection-associated cancers in the year 2002," International Journal of Cancer, vol. 118, no. 12, pp. 3030-3044, 2006.

[71] D. M. Parkin, P. Srivatanakul, M. Khlat et al., "Liver cancer in Thailand. I. A case-control study of cholangiocarcinoma," International Journal of Cancer, vol. 48, no. 3, pp. 323-328, 1991. 
[72] M. Haswell-Elkins, E. Miarang, P. Mairang et al., "Cross-sectional study of Opisthorchis viverrini infection and cholangiocarcinoma in communities within a high-risk area in Northeast Thailand," International Journal of Cancer, vol. 59, no. 4, pp. 505-509, 1994.

[73] S. Honjo, P. Srivatanakul, H. Sriplung et al., "Genetic and environmental determinants of risk for cholangiocarcinoma via Opisthorchis viverrini in a densely infested area in Nakhon Phanom, Northeast Thailand," International Journal of Cancer, vol. 117, no. 5, pp. 854-860, 2005.

[74] B. I. Choi, J. K. Han, S. T. Hong, and K. H. Lee, "Clonorchiasis and cholangiocarcinoma: etiologic relationship and imaging diagnosis," Clinical Microbiology Reviews, vol. 17, no. 3, pp. 540552, 2004.

[75] M. R. Haswell-Elkins, S. Satarug, M. Tsuda et al., "Liver fluke infection and cholangiocarcinoma: model of endogenous nitric oxide and extragastric nitrosation in human carcinogenesis," Mutation Research-Fundamental and Molecular Mechanisms of Mutagenesis, vol. 305, no. 2, pp. 241-252, 1994. 


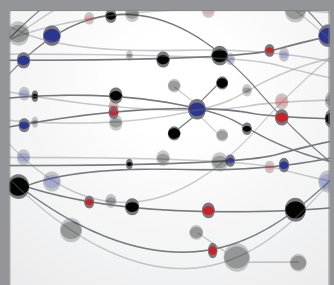

The Scientific World Journal
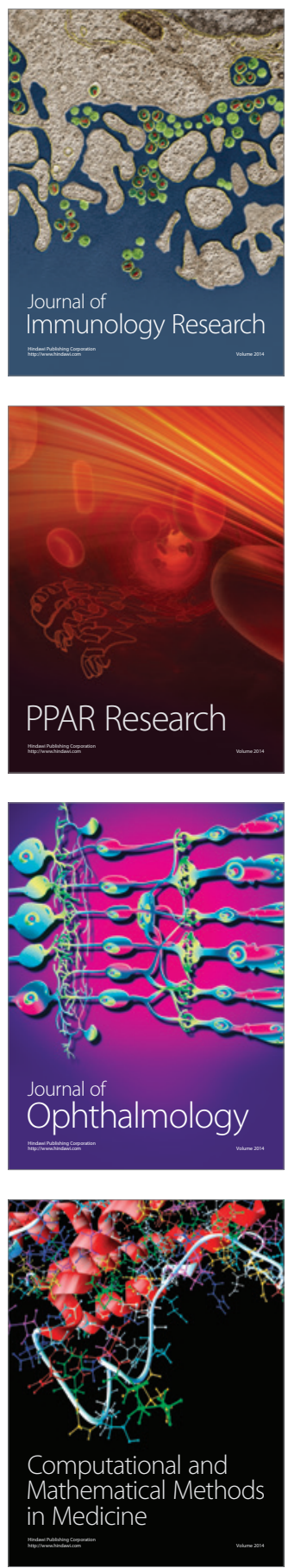

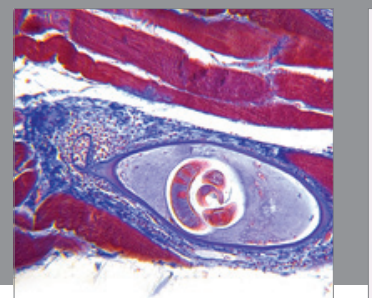

Gastroenterology

Research and Practice
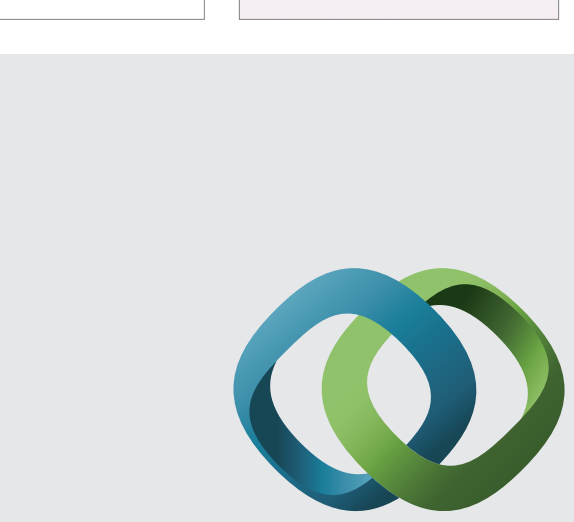

\section{Hindawi}

Submit your manuscripts at

http://www.hindawi.com
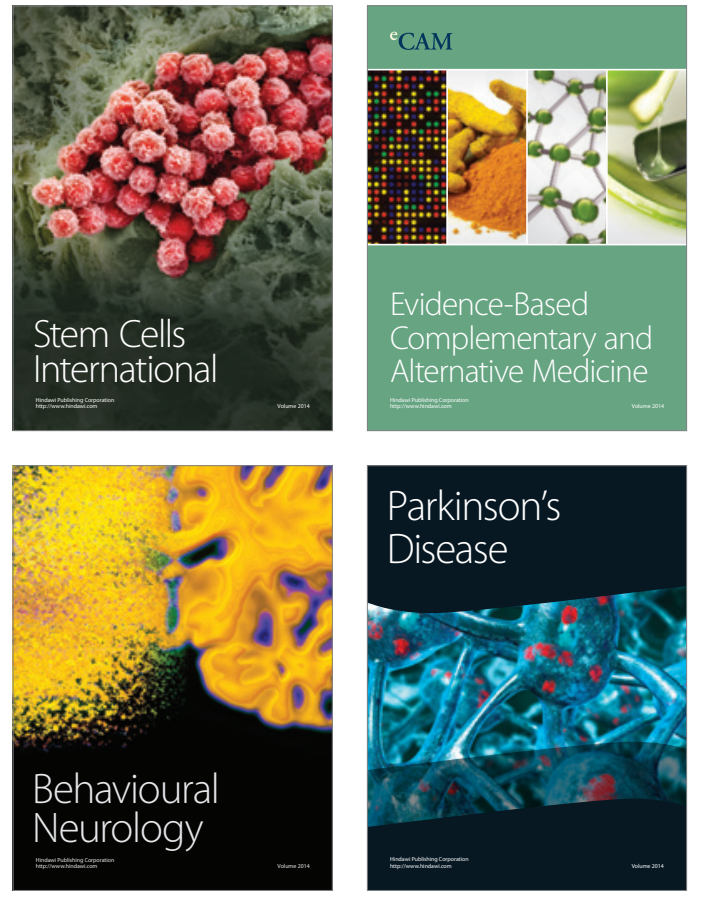
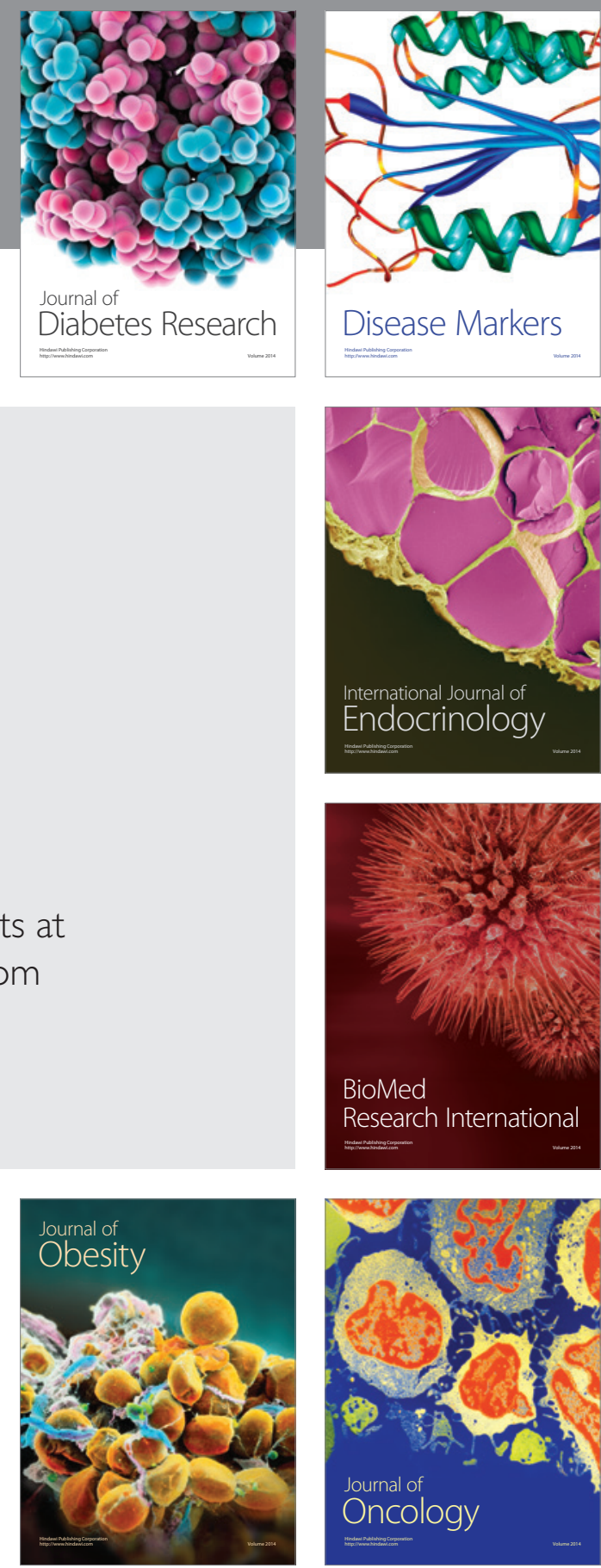

Disease Markers
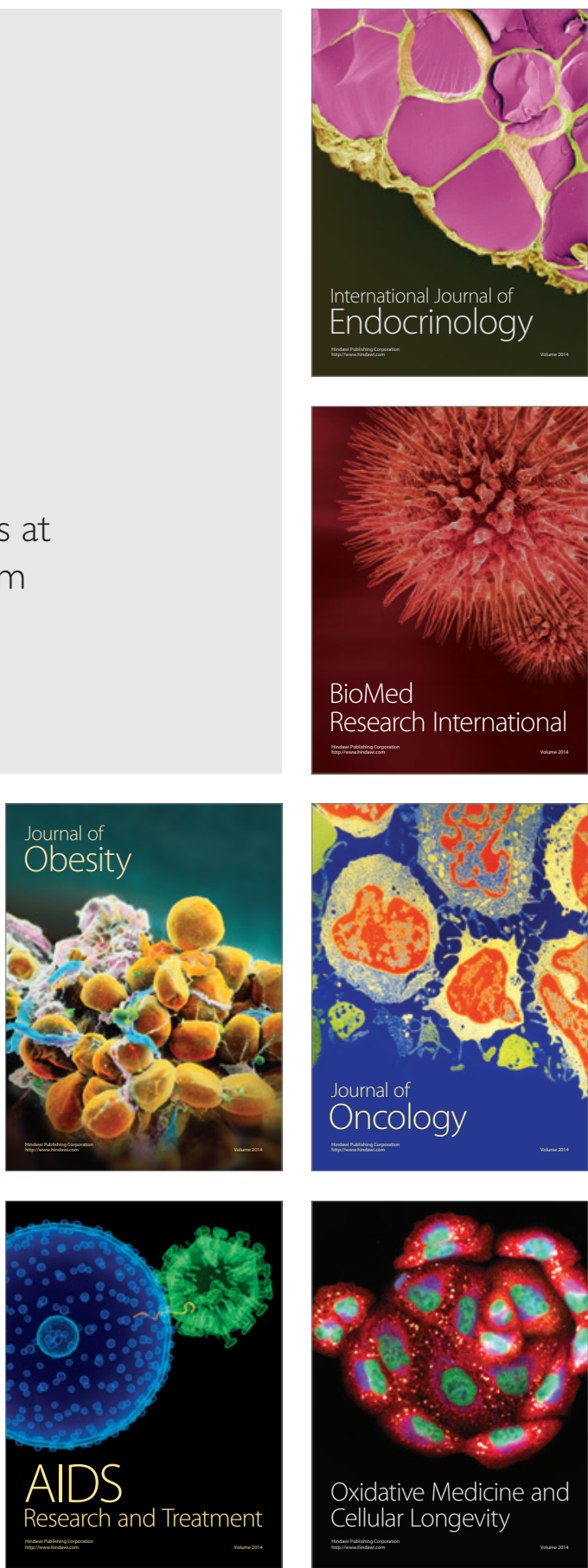